\title{
Monte Carlo Investigation of Blume-Emery-Griffiths Model with Mixed Spins
}

\author{
M. Bourass ${ }^{a}, \mathrm{~A} \mathrm{ZRAdba}^{a}, \mathrm{~A} \cdot \mathrm{Al}-\mathrm{RAJHi}^{b}, \mathrm{~N} \mathrm{HaChem}^{a}$, \\ R. Aharrouch ${ }^{a}$, M. Madani ${ }^{c}$ And M. El Bouziani ${ }^{a, *}$ \\ ${ }^{a}$ Team of Theoretical Physics, Laboratory L.P.M.C., Faculty of Sciences, \\ Chouaib Doukkali University, El Jadida, Morocco \\ ${ }^{b}$ Department of Information Technologies, Faculty of Education and Applied Sciences, \\ Hodeidah University, Raymah, Yemen \\ ${ }^{c}$ Department of Physics and Chemistry, CRMEF, Meknes, Morocco
}

Received: 26.08.2021 \& Accepted: 19.11.2021

Doi: 10.12693/APhysPolA.141.47

*e-mail: elbouziani.m@ucd.ac.ma

\begin{abstract}
Using Monte Carlo simulation, we have investigated the phase diagrams and magnetic properties of a mixed spin- 1 and spin-3/2 Blume-Emery-Griffiths model on a simple cubic lattice. First, the thermal variations of the magnetizations of the Blume-Capel model (Blume-Emery-Griffiths model with zero biquadratic coupling) as well as its phase diagram in the (temperature, crystal field) plane are plotted showing the second-order phase transitions and the compensation temperatures. Then, we have studied the effect of the introduction of a biquadratic coupling on the critical and compensation behaviours already observed in the Blume-Capel model.
\end{abstract}

topics: mixed spins, Blume-Emery-Griffiths, Monte Carlo, phase diagram

\section{Introduction}

The Blume-Emery-Griffiths (BEG) model [1] is one of the important lattice-spin models in statistical physics for the analysis of the phenomenon of phase transitions [2]. This is a spin-1 Ising model with bilinear and biquadratic nearestneighbour couplings and crystal field interaction, originally introduced by Blume, Emery and Griffiths to simulate the thermodynamic behaviour of ${ }^{3} \mathrm{He}-{ }^{4} \mathrm{He}$ mixtures. Later, the BEG model was reinterpreted to describe the phase transitions and critical phenomena in many other systems, such as semiconductor alloys [3], solid-liquid-gas systems [4], microemulsions [5], electronic conduction models [6] etc.

Theoretically, the BEG model has been examined by several methods, namely mean-field approximation (MFA) [1,7-10], renormalization group (RG) techniques [7, 10-12], Monte Carlo simulation (MCS) $[8,11,13-15]$, the exact recursion equations on the Bethe lattice $[16,17]$ and the effective field theory (EFT) with correlations [18]. Most of these works exhibit a rich variety of phase diagrams showing second and first order transitions, critical endpoints, multi-critical points and reentrant behaviour. Moreover, the presence of a non-zero biquadratic interaction $(K \neq 0)$ results in the widening of the first-order transitions domain for $K>0$, while there is a clear reduction in this domain before vanishing for $K<0$.

In recent years, remarkable attention has been given to mixed-spin systems because of their interesting properties for studying ferrimagnetism [19], magneto-optical recording materials [20] and information storage devices [21]. These mixed systems consist of two interpenetrating and non-equivalent sublattices with two unequal magnetic moments. Consequently, many new phenomena appear that cannot be observed in pure-spin systems, e.g. the compensation temperatures. At these last points, the total magnetization vanishes but the system has not yet reached the critical temperature. This is explained by the fact that the magnetizations of the two sublattices are ferrimagnetically coupled and have equal magnitude but opposite sign. For instance, the oxalato-bridged heterotrinuclear complex $\left.\left[\mathrm{NiCr}_{2} \text { (bipy) }\right)_{2}\left(\mathrm{C}_{2} \mathrm{O}_{4}\right)_{4}\left(\mathrm{H}_{2} \mathrm{O}\right)_{2}\right] \mathrm{H}_{2} \mathrm{O}[22]$ is a good example of a mixed-spin system, and which exhibits a ferrimagnetic coupling between $\mathrm{Ni}(\mathrm{II})$ with $S=1$ and $\operatorname{Cr}($ III) with $S=3 / 2$.

Among the mixed-spin systems recently studied by researchers is the mixed spin- 1 and spin-3/2 BEG model. It has been investigated by MCS on 
a honeycomb lattice [23] and by the two methods MFA and Migdal-Kadanoff RG technique on a hypercubic lattice with attractive and repulsive biquadratic couplings [10, 24]. These three mentioned works show various types of phase diagrams with first and second order transitions, tricritical and critical endpoints as well as compensation behaviour according to different physical parameters: bilinear $(J)$ and biquadratic $(K)$ interactions, crystal field and external magnetic field.

Thus, since the results of the Monte Carlo simulation are more reliable compared to other approximation techniques, we have planned the investigation of the mixed spin- 1 and spin-3/2 Blume-EmeryGriffiths model on a simple cubic lattice using MCS in order to confirm the results obtained by other methods $[10,24]$ in the absence of exact solutions, in particular the domain of $K$ which favors the critical and compensation points.

We organize our paper as follows: in Sect. 2, we define the model to be studied and briefly give the MCS for our system. The phase diagrams and the numerical results are presented and discussed in Sect. 3. Finally, we provide conclusion in Sect. 4.

\section{Model and Monte Carlo simulation}

We consider a ferrimagnetic BEG model with mixed spins $S=3 / 2$ and $\sigma=1$ located in alternating sites of a simple cubic lattice. The two sublattices $(A)$ and $(B)$ are occupied, respectively, by spins $S_{i}$ which can take the values of $\pm 3 / 2$ and $\pm 1 / 2$, and by spins $\sigma_{j}$ which can take the values of \pm 1 and 0 . The Hamiltonian of this system is given by [1]

$$
\begin{aligned}
H & =-J_{A B} \sum_{\langle i j\rangle} S_{i} \sigma_{j}-K \sum_{\langle i j\rangle} S_{i}^{2} \sigma_{j}^{2} \\
& -\Delta\left(\sum_{i \in(A)} S_{i}^{2}+\sum_{j \in(B)} \sigma_{j}^{2}\right),
\end{aligned}
$$

where $J_{A B}$ and $K$ are the bilinear and biquadratic interactions, respectively, between the first nearest neighbouring spins $\langle i j\rangle\left(J_{A B}<0\right.$ since we study the ferrimagnetic case), and $\Delta$ is the crystal field applied on the spins $S$ and $\sigma$.

In this work, we simulate the Hamiltonian described by (1) on a simple cubic lattice of volume $L \times L \times L$ with a linear lattice size $L=30$ using the Monte Carlo method based on the Metropolis algorithm [25, 26]. Beginning from a random initial configuration of spins, our program generates configurations by trying to flip the spins - these flips are accepted or rejected according to a probability in Boltzmann statistics. Moreover, we apply periodic boundary conditions in the three directions. At each temperature, the averages of the physical quantities are calculated using $10^{5}$ Monte Carlo steps per site after discarding the first $2 \times 10^{4}$ steps per site to reach the equilibrium of the system. It is also interesting to mention the existence of perturbations in the Monte Carlo simulation for systems with spins larger than $\frac{1}{2}$, notably in the regions separating the ordered and disordered phases at low temperatures.

The magnetizations per site $m_{A}$ and $m_{B}$ of the sublattices $(A)$ and $(B)$ are expressed, respectively, as

$$
m_{A}=\frac{2}{N} \sum_{i \in(A)} S_{i},
$$

and

$$
m_{B}=\frac{2}{N} \sum_{j \in(B)} \sigma_{j}
$$

and the total magnetization per site $M_{\mathrm{T}}$ is defined by

$$
M_{\mathrm{T}}=\frac{m_{A}+m_{B}}{2},
$$

where $N=L \times L \times L$ is the number of magnetic atoms of the system, and $\beta=\left(k_{\mathrm{B}} T\right)^{-1}$ with $k_{\mathrm{B}}$ the Boltzmann constant and $T$ the absolute temperature. In the following, we take $k_{\mathrm{B}}=1$.

\section{Results and discussion}

\subsection{Case of $K=0$ (Blume-Capel model)}

First of all, it should be noted that the BlumeCapel (BC) model [27, 28] is a BEG system with zero biquadratic interaction $(K=0)$. Let's start by presenting our results concerning the $\mathrm{BC}$ model with mixed spins $(1,3 / 2)$, in particular the influence of the crystal field $\Delta$ on the magnetic properties of the system. Figures 1 and 2 show, respectively, the temperature dependence of the sublattice magnetizations $\left(m_{A}\right.$ and $\left.m_{B}\right)$ and the total magnetization $\left(M_{\mathrm{T}}\right)$ for some selected values of $\Delta /\left|J_{A B}\right|=-3.0,-2.99,-2.95,-2.90,-2.50,-2.0$ and -1.0 when $K=0$.

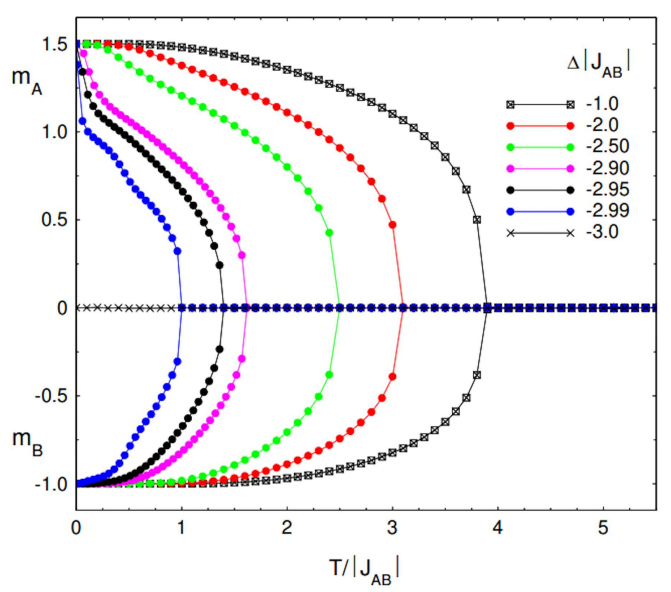

Fig. 1. The thermal variations of the sublattice magnetizations per site $m_{A}$ and $m_{B}$ for some selected values of $\Delta /\left|J_{A B}\right|$ when $K=0$. 


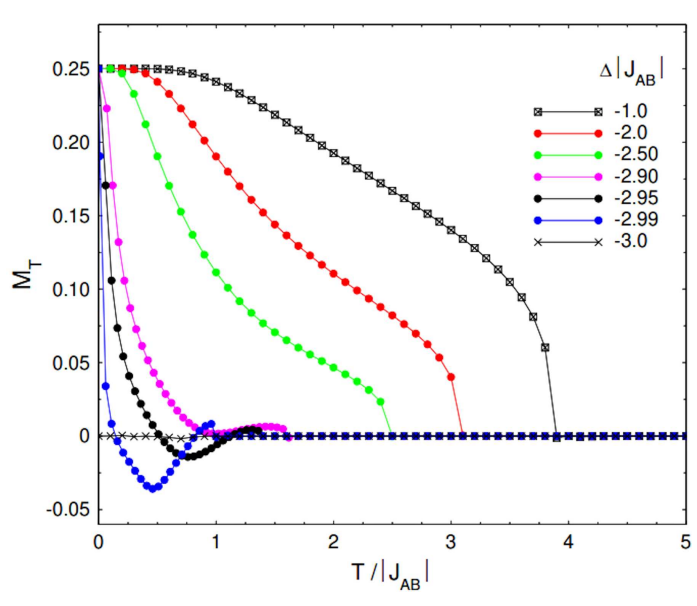

Fig. 2. The thermal variations of the total magnetization per site $M_{\mathrm{T}}$ for some selected values of $\Delta /\left|J_{A B}\right|$ when $K=0$.

In Fig. 1, when the temperature increases, one can observe that for all selected values of $\Delta /\left|J_{A B}\right|$, except for -3.0 , the magnetization $m_{A}$ decreases continuously from its saturation value 1.5 at $T=0$ until vanishing beyond the critical temperature $T_{C}$, whereas the magnetization $m_{B}$ starts from its minimum value -1.0 and increases in a continuous way to zero at $T_{C}$. This justifies that there is only a second-order phase transition for these values of $\Delta /\left|J_{A B}\right|$. However, for $\Delta /\left|J_{A B}\right|=-3.0, m_{A}$ and $m_{B}$ are zero regardless of temperature.

In Fig. 2, by increasing the temperature for $\Delta /\left|J_{A B}\right|=-2.50,-2.0$ and -1.0 , the total magnetization $M_{\mathrm{T}}$ decreases continuously from its saturation value 0.25 at $T=0$, to vanish beyond $T_{C}$. For $\Delta /\left|J_{A B}\right|=-2.90,-2.95$ and $-2.99, M_{\mathrm{T}}$ decreases monotonically from the saturation value 0.25 to a minimum, then increases to a slightly greater value than 0 , and finally decreases to zero at $T_{C}$. In the case of $\Delta /\left|J_{A B}\right|=-2.95$ and -2.99 as opposed to $\Delta /\left|J_{A B}\right|=-2.90$, the minimum value of $M_{\mathrm{T}}$ is smaller than 0 , which means that $M_{\mathrm{T}}$ meets the axis $M_{\mathrm{T}}=0$ at two points with temperatures lower than $T_{C}$, and these are compensation temperatures $\left(T_{\text {comp }}\right)$. Finally, for $\Delta /\left|J_{A B}\right|=-3.0, M_{\mathrm{T}}$ is null for all values of temperature. Our system presents here five types of $M_{\mathrm{T}}$ curves according to the extended Néel's nomenclature $[29,30]$, i.e., types Q, R, S, N and $\mathrm{W}$.

In order to clearly show the compensation behaviour, we present the thermal variations of $M_{\mathrm{T}}$ on a smaller scale in the vicinity of the compensation points for $\Delta /\left|J_{A B}\right|=-2.99,-2.97,-2.95,-2.93$, $-2.91,-2.90$ as plotted in Fig. 3. The total magnetization $M_{\mathrm{T}}$ exhibits two compensation points in the range $-2.99 \leq \Delta /\left|J_{A B}\right| \leq-2.91$, while there are no compensation points for $\Delta /\left|J_{A B}\right| \geq-2.90$.

Figure 4 displays the phase diagram in the $\left(\Delta /\left|J_{A B}\right|, T /\left|J_{A B}\right|\right)$ plane, when $K=0$, plotted after determining the critical and compensation

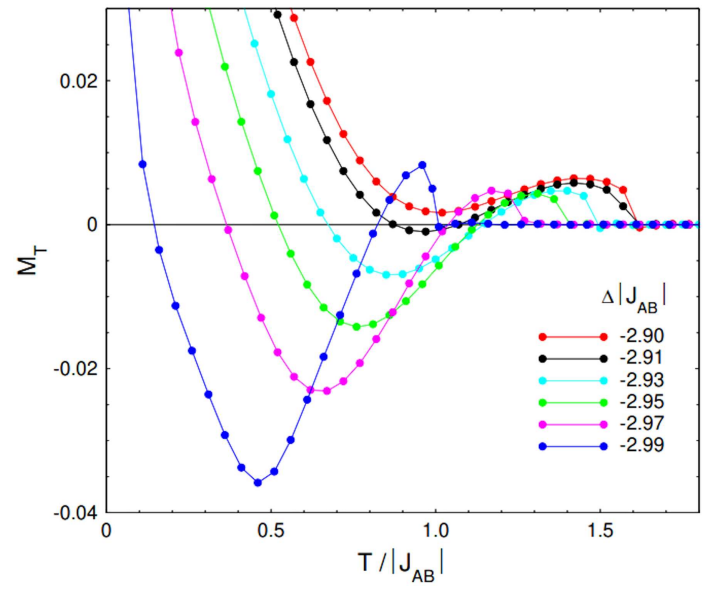

Fig. 3. The thermal variations of the total magnetization per site $M_{\mathrm{T}}$ on a smaller scale in the vicinity of the compensation points for some selected values of $\Delta /\left|J_{A B}\right|$ when $K=0$.

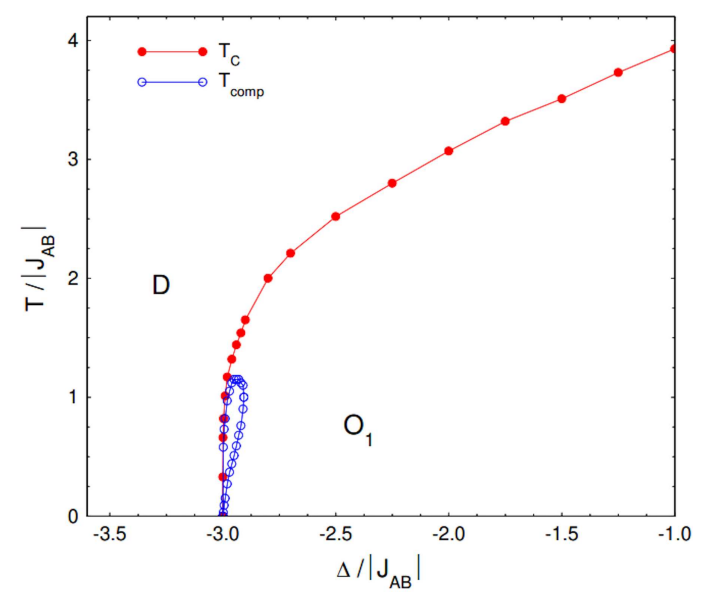

Fig. 4. The phase diagram of the system in the $\left(\Delta /\left|J_{A B}\right|, T /\left|J_{A B}\right|\right)$ plane when $K=0$.

temperatures for several values of $\Delta /\left|J_{A B}\right|$. Looking at this phase diagram, it is shown that there is a second-order phase transition separating the ferrimagnetic ordered phase $O_{1} \equiv\left(m_{A}=\right.$ $\left.3 / 2, m_{B}=-1\right)$ from the paramagnetic disordered phase $D \equiv\left(m_{A}=0, m_{B}=0\right)$ and starting from $\left(\Delta /\left|J_{A B}\right|=-3.0, T=0\right)$. Moreover, when $\Delta /\left|J_{A B}\right|$ increases, the critical temperature increases up to a constant value for large values of the crystal field. In the ferrimagnetic phase domain for $-3.0<$ $\Delta /\left|J_{A B}\right| \leq-2.905$ we see a curve of compensation points, such that for each $\Delta /\left|J_{A B}\right|$ belongs to this range there are two compensation points.

Our results obtained in this study can be compared with those found for Ising systems with mixed spins (1, 3/2) using MCS [31, 32]. Nakamura and Tucker have examined the magnetic properties of an Ising ferromagnetic system with these mixed spins on a cubic lattice [31]. They have found 
first-order transitions but did not find compensation points since they took into account the ferromagnetic case $\left(J_{A B}>0\right)$. Also, Žukovič and Bobák have investigated a mixed spin-1 and spin$3 / 2$ Ising ferrimagnet on a square lattice [32], and they have reported the presence of multicompensation behaviour, as we did - our phase diagram confirms this plotted in the last study.

We should also mention that other approaches studying BC systems with equal crystal field for the sites of spin- 1 and spin- $3 / 2$ validate our results. For instance, the formulation of random crystal field on the Bethe lattice [33] and the MFA [34, 35] exhibit a multicompensation behaviour. However, the exact recursion equations on the Bethe lattice [36], the cluster variational theory within pair approximation [37] and the MFA [34, 35] show the firstorder transition lines within the ferrimagnetic ordered region. Moreover, in most of these works [3437 , the system presents tricritical points and transitions (order-disorder) of first-order, which is inconsistent with us.

\subsection{BEG phase diagrams}

In this subsection, we introduce the non-zero biquadratic interaction $(K \neq 0)$ into the system and examine its influence on the magnetic properties and the phase diagram of the model, as well as the effect of the crystal field in the presence of $K \neq 0$.

In Figs. 5 and 6 we present, respectively, the sublattice magnetizations and the total magnetization as a function of temperature for chosen values of $K /\left|J_{A B}\right|=0.0,-0.1,-0.3,-0.4,-0.6,-0.9$ and -0.99 when $\Delta /\left|J_{A B}\right|=-1.5$. In Fig. 5 , one sees that for all the presented values of $K /\left|J_{A B}\right|$ the magnetizations $m_{A}$ and $m_{B}$ vary continuously $\left(m_{A}\right.$ decreases and $m_{B}$ increases) from their saturation values at $T=0$, to vanish beyond $T_{C}$. The saturation values of $\left(m_{A}\right.$ and $\left.m_{B}\right)$ are respectively (1.5 and -1.0$)$ for $K /\left|J_{A B}\right|=0.0$, and -0.1 , and $(0.5$ and -1.0$)$ for $K /\left|J_{A B}\right|=-0.3,-0.4,-0.6$. We report that $m_{B}$ has saturation values between -1.0 and -0.5 for $-1.0<K /\left|J_{A B}\right| \leqslant-0.9$. This means that there is only a second-order phase transition, as well as the existence of two ferrimagnetic ordered phases, namely $O_{1} \equiv\left(m_{A}=3 / 2, m_{B}=-1\right)$ and $O_{2} \equiv\left(m_{A}=1 / 2, m_{B}=-1\right)$. The absence of a first-order transition between these two coexisting ordered phases has been verified.

With regard to the total magnetization curves plotted in Fig. 6, it is observed that for two values of $K /\left|J_{A B}\right|=0.0$ and $-0.1, M_{\mathrm{T}}$ decreases continuously from its initial value 0.25 at $T=0$ to zero at $T_{C}$, while for the other values of $K /\left|J_{A B}\right|$, $M_{\mathrm{T}}$ grows from the saturation value between 0.0 and -0.25 until it vanishes beyond $T_{C}$. However, for $K /\left|J_{A B}\right|=-0.3,-0.4,-0.9$ and $-0.99, M_{\mathrm{T}}$ shows a single compensation temperature, while for $K /\left|J_{A B}\right|=-0.6$, this temperature is absent.

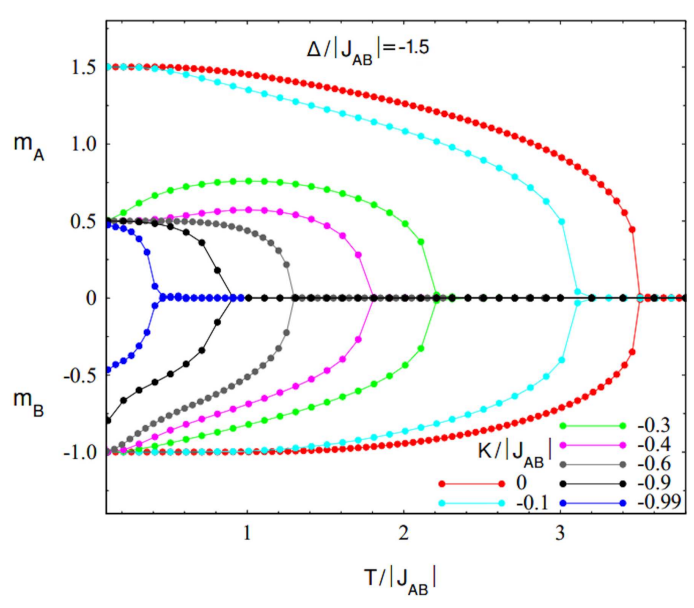

Fig. 5. The thermal variations of the sublattice magnetizations per site $m_{A}$ and $m_{B}$ for some selected values of $K /\left|J_{A B}\right|$ when $\Delta /\left|J_{A B}\right|=-1.5$.

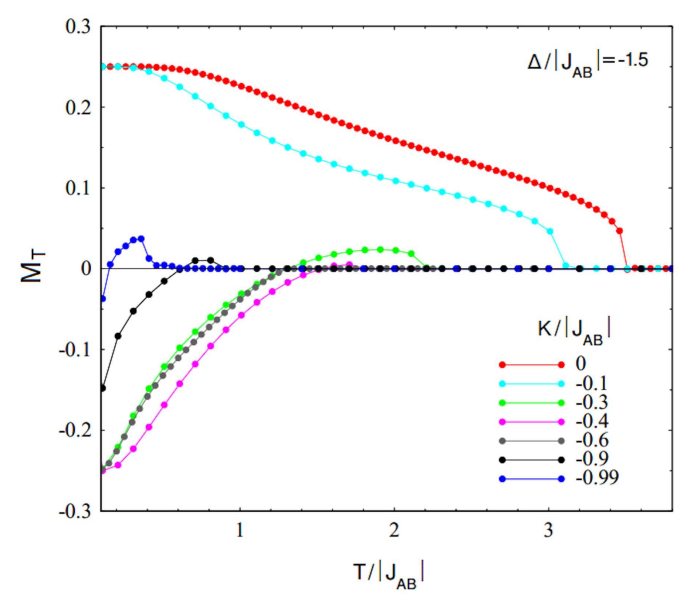

Fig. 6. The thermal variations of the total magnetization per site $M_{\mathrm{T}}$ for some selected values of $K /\left|J_{A B}\right|$ when $\Delta /\left|J_{A B}\right|=-1.5$.

In Fig. 7, a phase diagram is plotted in the $\left(K /\left|J_{A B}\right|, T /\left|J_{A B}\right|\right)$ plane for $\Delta /\left|J_{A B}\right|=-1.5$. First, one notices that the model exhibits two ferrimagnetic ordered phases $\left(\mathrm{O}_{1}\right.$ and $\left.\mathrm{O}_{2}\right)$ and a paramagnetic disordered $(D)$ phase. Second, there is a second-order phase transition that separates the ordered phases from the disordered phase. Third, two compensation lines appear in the $\left(\mathrm{O}_{2}\right)$ region, i.e., the first is situated in the interval $-0.99 \leq$ $K /\left|J_{A B}\right| \leq-0.79$ and limited by two extremities where it meets the critical second-order line, and the other is in the interval $-0.43 \leq K /\left|J_{A B}\right| \leq-0.25$ with two isolated endpoints far from the secondorder line. As a comparison, similar phase diagrams have been obtained in $[15,16,38]$ for BEG systems with other mixed spins using the exact recursion equations on the Bethe lattice for values of $\Delta /\left|J_{A B}\right|$ close to our value $\Delta /\left|J_{A B}\right|=-1.5$. The disagreement with the two papers $[16,38]$, however, is that they show only one compensation line in the ordered domain. 


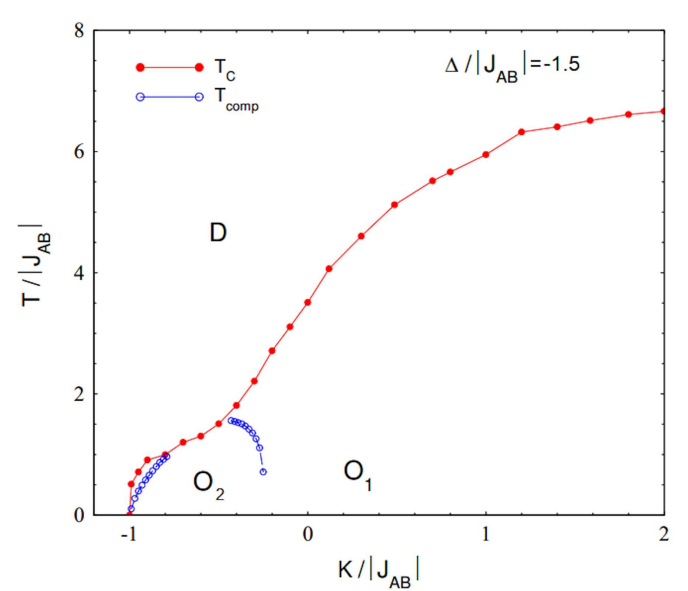

Fig. 7. The phase diagram of the system in the $\left(K /\left|J_{A B}\right|, T /\left|J_{A B}\right|\right)$ plane when $\Delta /\left|J_{A B}\right|=-1.5$.

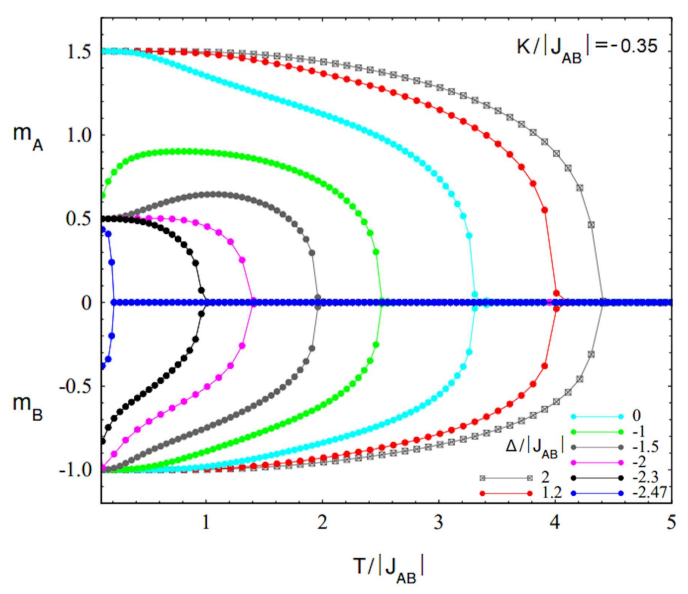

Fig. 8. The thermal variations of the sublattice magnetizations per site $m_{A}$ and $m_{B}$ for selected values of $\Delta /\left|J_{A B}\right|$ when $K /\left|J_{A B}\right|=-0.35$.

By comparing our results with those found in two papers [10, 24], which investigated the same BEG model with mixed spins $(1,3 / 2)$ by the MFA and RG method, we can notice that the appearance of compensation temperatures for negative values of $K$ in $[10]$ is in accordance with our phase diagram obtained in Fig. 7. The MCS does not give compensation points for $K>0$, although there are always critical points; the compensation domain is limited to $K<0$, where two compensation points were observed using MFA [10] and only one point by MCS. Certainly, the MCS results are more credible than those of the MFA, where the dimension is omitted and only the symmetry is taken into account via the number of first nearest neighbours. Furthermore, the absence of first-order transitions is in agreement with the predictions of the renormalization (for $K /\left|J_{A B}\right|<-0.5$ ) [10], while they are always present for $K<0$ by MFA [10] and for $K>0$ by RG [24].

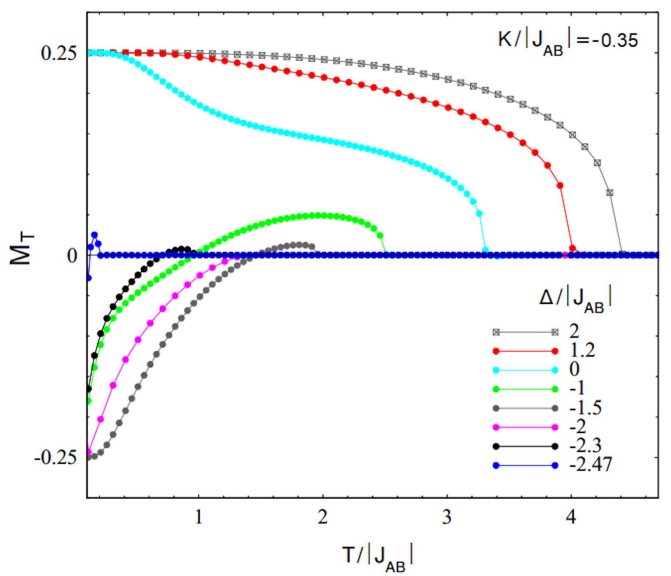

Fig. 9. The thermal variations of the total magnetization per site $M_{\mathrm{T}}$ for selected values of $\Delta /\left|J_{A B}\right|$ when $K /\left|J_{A B}\right|=-0.35$.

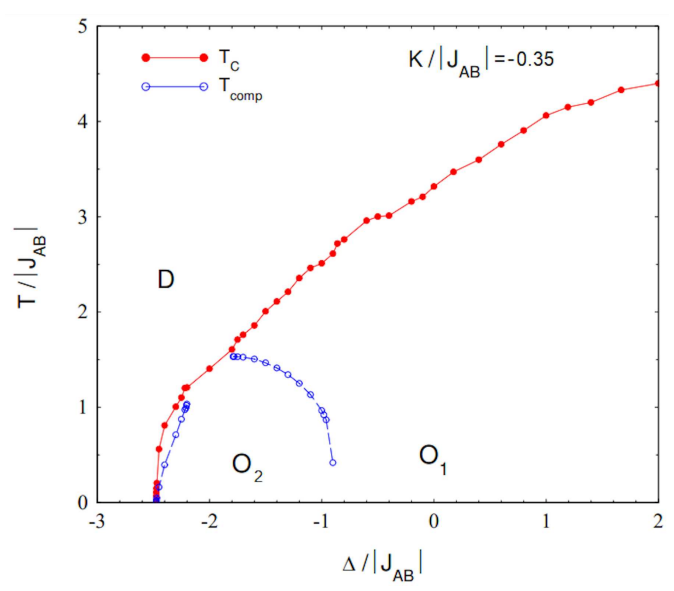

Fig. 10. The phase diagram of the system in the $\left(\Delta /\left|J_{A B}\right|, T /\left|J_{A B}\right|\right)$ plane when $K /\left|J_{A B}\right|=-0.35$.

Let us analyze the influence of the presence of $K \neq 0$ on the critical behaviour observed in the $\mathrm{BC}$ model $(K=0)$ for $\Delta /\left|J_{A B}\right|=-1.5$, where the system presents only $T_{C}$ describing the second-order transition. The first finding is the appearance of a new ordered phase $O_{2} \equiv\left(m_{A}=1 / 2, m_{B}=-1\right)$ for values of $K<0$. Also, by increasing $K>0$, $T_{C}$ increases up to saturation for large values of $K$, but for $K<0$ the compensation points $T_{\text {comp }}$ appear for some values and the critical temperature decreases with the decrease of $K$ until the disappearance at $K=-1.0$. However, we note the absence of first-order transition lines in the ordered region whatever $K \neq 0$.

Now, the effect of the crystal field on the magnetic properties of the system for $K \neq 0$ is investigated in Figs. 8-10. Foremost, the thermal variations of the sublattice and the total magnetizations are presented in Figs. 8 and 9, respectively, for a fixed value of $K /\left|J_{A B}\right|=-0.35$ and several selected values of $\Delta /\left|J_{A B}\right|=2.0,1.2,0.0,-1.0,-1.5,-2.0$, -2.3 and -2.47 . The first observation from these 
two figures is that when $\Delta /\left|J_{A B}\right|>-0.8$ as for $\Delta /\left|J_{A B}\right|=2.0,1.2$ and 0.0 , the saturation values of $m_{A}, m_{B}$ and $M_{\mathrm{T}}$ at $T=0$ are respectively $1.5,-1.0$ and 0.25 , and so the stable ordered phase in this region is $O_{1} \equiv\left(m_{A}=3 / 2, m_{B}=-1\right)$. In turn, when $-2.2<\Delta /\left|J_{A B}\right|<-0.8$ as for $\Delta /\left|J_{A B}\right|=-1.0$, -1.5 and -2.0 , the saturation values of $m_{A}, m_{B}$ and $M_{\mathrm{T}}$ at $T=0$ are respectively $0.5,-1.0$ and -0.25 , and so the stable ordered phase in this region is $O_{2} \equiv\left(m_{A}=1 / 2, m_{B}=-1\right)$. It should be noted that for $-2.47 \leqslant \Delta /\left|J_{A B}\right| \leq-2.3$, the saturation value of $m_{B}$ is relatively greater than -1 . The second observation is that all the curves of $m_{A}, m_{B}$ and $M_{\mathrm{T}}$ vary continuously from their saturation values at $T=0$ until they vanish beyond $T_{C}$, thus the system only shows the second-order transition here. Finally, it is necessary to point out the existence of compensation temperatures in Fig. 9 for certain values of $\Delta /\left|J_{A B}\right|$, especially for $\Delta /\left|J_{A B}\right|=-1.0$, $-1.5,-2.3$ and -2.47 .

Figure 10 exhibits the phase diagram in the $\left(\Delta /\left|J_{A B}\right|, T /\left|J_{A B}\right|\right)$ plane for $K /\left|J_{A B}\right|=-0.35$. One can observe that there are two ordered phases $\left(\mathrm{O}_{1}\right.$ and $\left.\mathrm{O}_{2}\right)$ at low temperatures separated from the disordered phase $(D)$ by a second-order transition line. We also highlight the existence of two compensation lines in the $\left(\mathrm{O}_{2}\right)$ region as in Fig. 7, located precisely in the two ranges $-2.47 \leq$ $\Delta /\left|J_{A B}\right| \leq-2.20$ and $-1.79 \leq \Delta /\left|J_{A B}\right| \leq-0.90$. In addition, the system does not give any first-order transition line in the ordered domain. This phase diagram resembles those found for BEG mixed-spin systems using exact recursion relations on the Bethe lattice in $[16,17]$. One also notices the presence of the compensation behaviour for certain values of $\Delta /\left|J_{A B}\right|$ when $K \neq 0$ in the BEG model with mixed spins $(1,3 / 2)$ by MFA [10] and MCS [23] as we have already shown.

\section{Conclusion}

In summary, the ferrimagnetic mixed spin-1 and spin-3/2 Blume-Emery-Griffiths model is studied using Monte Carlo simulation. Firstly, we have plotted the phase diagram of the Blume-Capel (zero biquadratic interaction $K=0$ ) where we have found the second-order phase transition and multicompensation behaviour. Secondly, we have introduced the $K \neq 0$ values into the model and we have studied the influence of this on the magnetic properties of the system. The plotted phase diagrams show the existence of a new ferrimagnetic ordered phase $\left(\mathrm{O}_{2}\right)$ for $K<0$, where two compensation lines appear in this region. Our results are consistent with those obtained in other literature studies.

\section{References}

[1] M. Blume, V.J. Emery, R.B. Griffths, Phys. Rev. A 4, 1071 (1971).

[2] C.K. Hu, Chin. J. Phys. 52, 1 (2014).
[3] K.E. Newman, J.D. Dow, Phys. Rev. B 27. $7495,1983)$.

[4] J. Lajzerowicz, J. Sivardiére, Phys. Rev. A 11, 2079 (1975).

[5] M. Schick, W.H. Shih, Phys. Rev. B 34, 1797 (1986).

[6] S.A. Kivelson, V.J. Emery, H.Q. Lin, Phys. Rev. B 42, 6523 (1990).

[7] A.N. Berker, M. Wortis, Phys. Rev. B 14, 4946 (1976).

[8] F.C. Sá Barreto, O.F. De Alcantara Bonfim, Physica A 172, 378 (1991).

[9] A. Bakchich, M. El Bouziani, Phys. Rev. B 56, 11161 (1997).

[10] A. Alrajhi, N. Hachem, A. Lafhal, A. El Antari, M. El Bouziani, M. Madani, M. El Falaki, J. Adv. Phys. 7, 261 (2018).

[11] N.S. Branco, Physica A 232, 477 (1996).

[12] A. Bakchich, M. El Bouziani, J. Phys. Condens. Matter 13, 91 (2001).

[13] A. Rachadi, A. Benyoussef, . Phys. Rev. B 69, 064423 (2004).

[14] S. Sidi Ahmed, L. Bahmad, A. Benyoussef, A. El Kenz, Superlattices Microstruct. 109, 841 (2017).

[15] M. Žukovič, M. Jaščur, Acta Phys. Pol. A 126, 36 (2014).

[16] E. Albayrak, Physica A 375, 174 (2007).

[17] R.A. Yessoufou, S. Bekhechi, F. Hontinfinde, Eur. Phys. J. B 81, 137 (2011).

[18] T. Kaneyoshi, M. Jaščur, Phys. Lett. A 177, 172 (1993).

[19] O. Kahn, Molecular Magnetism VCH, New York, 1993.

[20] A. Winterstein, H. Akamatsu, D. Möncke, K. Tanaka, M.A. Schmidt, L. Wondraczek, Opt. Mater. Express 3, 184 (2013).

[21] G.A. Prinz, Science 282, 1660 (1998).

[22] N. Stanica, C.V. Stager, M. Cimpoesu, M. Andruh, Polyhedron 17, 1787 (1998).

[23] S. Sidi Ahmed, L. Bahmad, A. El Yousfi, A. Benyoussef, A. El Kenz, A.G. El Hachimi, Superlattices Microstruct. 123, 1 (2018).

[24] A. Lafhal, A. El Antari, N. Hachem, A. Al-Rajhi, R. Aharrouch, H. Saadi, M. Madani, M. El Bouziani, Int. J. Theor. Phys. 59, 1165 (2020).

[25] N. Metropolis, A.W. Rosenbluth, M.N. Rosenbluth, A.H. Teller, E. Teller, J. Chem. Phys. 21, 1087 (1953).

[26] D.P. Landau, K. Binder, A Guide to Monte Carlo Simulations in Statistical Physics, 3rd Ed, Cambridge University Press, Cambridge 2009. 
[27] M. Blume, Phys. Rev. 141, 517 (1966).

[28] H.W. Capel, Physica 32, 966 (1966).

[29] L. Néel, Ann. Phys. 12, 137 (1948).

[30] J. Strečka, Physica A 360, 379 (2006).

[31] Y. Nakamura, J.W. Tucker, IEEE Trans. Magn. 38, 2406 (2002).

[32] M. Žukovič, A. Bobák, Physica A 389, 5402 (2010).

[33] A. Yigit, E. Albayrak, J. Supercond. Nov. Magn. 30, 3103 (2017).

[34] O.F. Abubrig, D. Horváth, A. Bobák, M. Jaščur, Physica A 296, 437 (2001).
[35] S. Sabri, M. El Falaki, M. El Yadari, A. Benyoussef, A. El Kenz, Physica A 460, 210 (2016).

[36] E. Albayrak, Int. J. Mod. Phys. B 17, 1087 (2003).

[37] J.W. Tucker, J. Magn. Magn. Mater. 237, 215 (2001).

[38] M. Karimou, R. Yessoufou, F. Hontinfinde, World J. Condens. Matter Phys. 5, 187 (2015). 